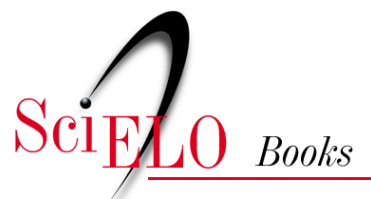

\title{
Sumário executivo
}

Comissão Nacional sobre Determinantes Sociais da Saúde

\section{SciELO Books / SciELO Livros / SciELO Libros}

COMISSÃO NACIONAL SOBRE DETERMINANTES SOCIAIS DA SAÚDE. Sumário executivo. In: As causas sociais das iniqüidades em saúde no Brasil [online]. Rio de Janeiro: Editora FIOCRUZ, 2008, pp. 193-204. ISBN: 978-85-7541-591-7. Available from: doi: 10.7476/9788575415917.0008. Also available in ePUB from: http://books.scielo.org/id/bwb4z/epub/comissao-9788575415917.epub.

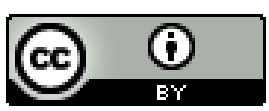

All the contents of this work, except where otherwise noted, is licensed under a Creative Commons Attribution 4.0 International license.

Todo o conteúdo deste trabalho, exceto quando houver ressalva, é publicado sob a licença $\underline{\text { Creative Commons }}$ Atribição 4.0. 


\section{SUMÁRIO EXECUTIVO}

\section{INTRODUÇÃo}

Em março de 2005, a Organização Mundial da Saúde (OMS) criou a Comissão sobre Determinantes Sociais da Saúde (Commission on Social Determinants of Health, CSDH), com o objetivo de promover, em âmbito internacional, uma tomada de consciência sobre a importância dos determinantes sociais na situação de saúde de indivíduos e populações e sobre a necessidade do combate às iniqüidades em saúde por eles geradas. Um ano depois, em 13 março de 2006, por meio de Decreto Presidencial, foi criada, no Brasil, a Comissão Nacional sobre Determinantes Sociais da Saúde (CNDSS), com um mandato de dois anos.

A CNDSS esteve integrada por 16 expressivas lideranças de nossa vida social, cultural, científica e empresarial. Sua constituição diversificada é uma expressão do reconhecimento de que a saúde é um bem público, construído com a participação solidária de todos os setores da sociedade brasileira.

Os objetivos da CNDSS podem ser assim resumidos:

- gerar informações e conhecimentos sobre os determinantes sociais da saúde no Brasil;

- contribuir para a formulação de políticas que promovam a eqüidade em saúde;

- mobilizar diferentes instâncias do governo e da sociedade civil sobre este tema.

Para alcançar seus objetivos, a CNDSS se apóia em três compromissos básicos:

Compromisso com a eqüidade: apesar dos importantes avanços dos últimos anos na melhoria do valor médio de seus indicadores de saúde, o Brasil está entre os países com maiores iniqüidades em saúde, ou seja, desigualdades de saúde entre grupos populacionais que além de sistemáticas e relevantes são também evitáveis, injustas e desnecessárias.

O compromisso da CNDSS com a eqüidade, visando a assegurar o direito universal à saúde, não é apenas uma decisão racional, mas, fundamentalmente, um compromisso ético e uma posição política.

Compromisso com a evidência: a CNDSS procura fundamentar suas análises e recomendações em sólidas evidências científicas, pois são estas que permitem, por um lado, entender como operam os determinantes sociais na geração das iniqüidades em saúde e, por outro, como e onde devem incidir as intervenções para combatê-las e que resultados podem ser esperados em termos de efetividade e eficiência. 
Compromisso com a ação: o compromisso maior da Comissão e que dá sentido à sua existência é o combate às iniqüidades em saúde por meio da atuação sobre os determinantes sociais que as geraram, os quais, sendo produto da ação humana, podem e devem ser modificados pela ação humana. O compromisso com a ação está alicerçado nas evidências científicas, conforme já assinalado, e numa ampla base de sustentação política, produto da conscientização e mobilização de diversos setores da sociedade.

Os diversos estudos sobre os DSS e as iniqüidades em saúde permitiram a construção de modelos que procuram esquematizar a trama de relações entre os vários níveis de determinantes sociais e a situação de saúde. Entre estes modelos, a CNDSS resolveu adotar o de Dahlgren e Whitehead (1991), que serve de base para orientar a organização de suas atividades e os conteúdos do presente relatório. A escolha se justifica por sua simplicidade, por sua fácil compreensão para vários tipos de público e pela clara visualização gráfica dos diversos DSS. Nesse modelo, os DSS estão dispostos em diferentes camadas, segundo seu nível de abrangência, desde uma camada mais próxima aos determinantes individuais até uma camada distal onde se situam os macrodeterminantes.

\section{Análise da Situação de Saúde}

\subsection{Situação e Tendências da Evolução Demográfica, Social e Econômica do País}

o Brasil vem passando por grandes transformações econômicas, sociais e demográficas, particularmente nas quatro últimas décadas, com significativas repercussões nas condições de vida e trabalho da população e, conseqüentemente, em sua situação de saúde.

O censo demográfico de 1960 revelava que 55\% da população economicamente ativa, portanto sua maioria, dedicavam-se à agricultura, enquanto os restantes $45 \%$ se dedicavam aos setores secundário e terciário. Já na década seguinte, essa proporção se inverte, com 54\% da população empregada na indústria ou no setor serviços. Segundo o último censo de 2000 , apenas $19 \%$ da população estavam empregados no campo, ou seja, uma queda de 55\% para $19 \%$ em quatro décadas. Nesse mesmo período, a população empregada pelo setor serviços passou de $27 \%$ a $60 \%$, enquanto que a dedicada à indústria, depois de uma ascensão de $17 \%$ a 29\% entre 1960 e 1980, caiu para $21 \%$ no censo de 2000.

Evidentemente, essa redistribuição acelerada da população economicamente ativa (PEA) do setor agrícola para os setores industriais e de serviços implicou um processo de urbanização também extraordinariamente acelerado. Em 1960, a maioria da população (55\%) possuía seu domicílio na zona rural. Na década seguinte, a proporção se inverteu, com 56\% da população residindo na área urbana, proporção que cresceu explosivamente desde então para atingir $81 \%$, em 2000.

Os processos de industrialização e urbanização acelerada foram responsáveis por importantes mudanças nos padrões de fecundidade da população. Segundo dados do Censo, a taxa média geométrica de crescimento anual da população passou de 2,89\%, no período 1960/1970, para 
1,64\% no período 1991/2000. A taxa de fecundidade, que era de 6,3 filhos por mulher em idade fértil em 1960, caiu para 2,3 em 2000, devendo situar-se em 2,0 em 2006, segundo projeções do IBGE. Embora a queda acelerada da taxa de fecundidade ocorra em todas as regiões do país, existem importantes diferenças segundo a escolaridade das mulheres. De acordo com dados da Pesquisa Nacional por Amostra de Domicílios (Pnad), de 2006, a taxa de fecundidade total que, em 2005, era de 2,1 filhos por mulher em idade fértil, variava de 4 para mulheres com até três anos de estudo a 1,5 para as que possuíam oito ou mais anos de estudo. Apesar das baixas taxas atuais de fecundidade, a população brasileira ainda deve crescer de maneira expressiva nas próximas décadas, como resultado da fecundidade passada. Haverá, também, uma importante modificação na estrutura etária, com envelhecimento da população, causado pela diminuição da fecundidade e aumento da expectativa de vida.

As quatro décadas entre 1960 e 2000 também foram marcadas por importantes transformações econômicas. Segundo o Ipeadata, o Produto Interno Bruto (PIB) per capita passou de 2.060 dólares, em 1960, para 5.250 em 2000 e 5.720 em 2006 (em valores constantes do dólar de 2006). A agropecuária, responsável por 25 \% do PIB em 1960, caiu sua participação para $8,9 \%$ em 2004, com um crescimento de 209\% nesse período, enquanto a indústria, que correspondia a $18 \%$ do PIB em 1960 , passou a responder por $42 \%$ do PIB em 2004 , com um crescimento de $1.727 \%$ no período.

Entretanto, esse extraordinário aumento da riqueza produzida e a modernização da economia não significaram melhoria importante na distribuição de renda. Mesmo com as melhorias recentes na distribuição de renda, relacionadas ao controle da inflação, à estabilidade macroeconômica (proporcionadas pelo Plano Real), à valorização do salário mínimo e aos programas de transferência de renda intensificados nos últimos anos, a distribuição de renda no Brasil continua entre as piores do mundo. Com base em um Índice de Gini de 0,57 em 2003, o relatório do Programa das Nações Unidas para o Desenvolvimento (Pnud), de 2007, situa o Brasil em $11^{\circ}$ lugar entre os países com mais alta concentração de renda (em 2006, o Índice de Gini caiu para 0,54).

$\mathrm{Na}$ esfera do desenvolvimento social, ocorreram também grandes mudanças nas últimas décadas, destacando-se, entre elas, as ocorridas na educação. Em 1940, 56\% da população brasileira era analfabeta, percentual que cai para 40\% no em 1960 e 13,6\% no ano 2000 . Segundo a Pnad, em 2006, havia 12,3\% de analfabetos entre as pessoas com 5 ou mais anos de idade, observando-se, entretanto, importantes diferenças regionais, já que este percentual na região Sul era de $7,6 \%$, enquanto no Nordeste era de $22,10 \%$. Há, também, importantes diferenças de acordo com a renda familiar. A média de analfabetos de 10,4\%, entre as pessoas com 15 ou mais anos de idade, variava de $17,9 \%$ para as pessoas com rendimento mensal familiar per capita menor que meio salário mínimo até 1,3\% para as pessoas com mais de dois salários mínimos.

Ainda segundo a Pnad 2006, há também extraordinário avanço da escolaridade no nível fundamental, com cobertura quase universal entre 7 a 14 anos, em todas as regiões, tanto na área urbana como rural. No caso do ensino médio, a taxa de freqüência líquida entre 15 
a 17 anos ainda é bastante baixa para o Brasil como um todo (cerca de 47\%), com grandes variações segundo região e segundo situação do domicílio (urbano/rural), enquanto para o ensino fundamental é de 95\%, sem grandes disparidades segundo estas variáveis.

Os importantes avanços e contradições no desenvolvimento econômico-social das últimas décadas são também observados na situação de saúde. Segundo o IBGE, a taxa de mortalidade infantil (TMI), que era de 124 óbitos no primeiro ano de vida para cada mil nascidos vivos em 1960, caiu para 48,3 em 1990; 35,26 em 2000 e 25,1 em 2006. Quanto à esperança de vida ao nascer, houve um ganho de mais de 20 anos, entre 1960 e 2006, para o Brasil como um todo, passando de 51,6 a 72,4. Embora persistam importantes diferenças regionais, como por exemplo, a expectativa de vida no Nordeste, em 2006, ainda é dois anos menor do que era a do Sul em 1990, há uma tendência à diminuição dessas diferenças. Em 1960, um brasileiro que nascesse no Nordeste tinha uma expectativa de vida 20 anos menor do que a de outro brasileiro nascido na região Sul, diferença essa que cai para cinco anos em 2006.

As tendências positivas observadas nas últimas décadas com relação à renda, escolaridade e saúde se expressam na evolução do índice de desenvolvimento humano (IDH), do PNUD, que passou de 0,649 em 1975 para 0,800 em 2005.

\subsection{A Estratificação Socioeconômica e a Saúde}

As condições socioeconômicas, culturais e ambientais de uma dada sociedade, ou seja, os determinates mais gerais e distais do modelo de Dahlgren e Whitehead, geram uma estratificação econômico-social dos indivíduos e grupos da população, conferindo-lhes posições sociais distintas, as quais por sua vez provocam diferenciais de saúde. Em outras palavras, a distribuição da saúde e da doença em uma sociedade não é aleatória, estando associada à posição social que, por sua vez, define as condições de vida e trabalho dos indivíduos e grupos.

Nesta seção, são apresentadas algumas associações entre estratificação socioeconômica (segundo renda, escolaridade, gênero, cor da pele e local de moradia) e resultados de saúde, destacando as iniqüidades em saúde derivadas da posição social ocupada por indivíduos e grupos da população.

A realização de exames preventivos para câncer de mama e de colo de útero, assim como de consultas pré-natal são bons exemplos das desigualdades de acesso e utilização de serviços de saúde, de acordo com escolaridade, observando-se um nítido gradiente segundo esta variável. A proporção de mulheres de 25 anos ou mais de idade que já realizaram alguma vez exame de mamografia varia de $24,3 \%$, para as mulheres sem instrução ou com menos de um ano de estudo, até $68,1 \%$ para as com 15 anos ou mais. A proporção de mulheres de 25 anos ou mais de idade que realizaram alguma vez exame preventivo para câncer de colo uterino varia de 55,8\%, para as com menos de um ano de estudo, até 93,1\% para as com 15 anos ou mais. Entre as mulheres com 12 ou mais anos de estudo, 20\% de seus filhos nasceram após um pré-natal com sete ou mais consultas, ao passo que para aquelas sem 
instrução esse percentual cai para 1,2\%. Inversamente, entre as mulheres sem instrução, $14,4 \%$ de seus filhos nasceram sem que houvesse nenhuma consulta pré-natal, porcentagem que cai para $2,7 \%$ entre as com 12 ou mais anos de estudo.

\subsection{Condições de Vida, Ambiente e Trabalho}

Neste item, apresentam-se alguns aspectos fundamentais das condições de vida e trabalho, com ênfase nas desigualdades existentes entre estas condições e sua associação com a situação de saúde. Destacam-se as condições de alimentação e nutrição, saneamento básico e habitação, condições de emprego e trabalho, ambiente e saúde, acesso a serviços de saúde e acesso à informação.

\section{Alimentação e Nutrição}

Nas últimas décadas, o Brasil vem passando por um processo de transição nutricional, que consiste na substituição de um padrão alimentar baseado no consumo de cereais, feijões, raízes e tubérculos por uma alimentação mais rica em gorduras e açúcares. Conforme ocorre com os processos de transição demográfia e epidemiológica, o processo de transição nutricional é também marcado pela sobreposição de padrões, pela temporalidade indefinida e, sobretudo, pelas desigualdades de acordo com a estratificação socioeconômica.

Essa mudança nos padrões alimentares vem aumentando o risco de sobrepeso e obesidade, condições que contribuem de forma importante para o aparecimento de doenças crônicas e incapacidades. A Pesquisa de Orçamento Familiar (POF), de 2003, mostrou que o número de brasileiros adultos com excesso de peso tinha praticamente dobrado em relação a 1974, quando foi feito o Estudo Nacional de Despesas Familiares. Em 2003, o excesso de peso atingia, em média, quatro em cada dez brasileiros adultos, superando em cerca de oito vezes o déficit de peso entre as mulheres e em quinze vezes entre os homens. Considerando o universo de brasileiros com 20 anos ou mais de idade, o IBGE estima que haja 3,8 milhões de pessoas (ou 4,0\%) com déficit de peso e 38,8 milhões (40,6\%) com excesso de peso, das quais 10,5 milhões são consideradas obesas.

\section{Saneamento Básico e Habitação}

A Pesquisa Nacional por Amostra de Domicílios (Pnad) registrou melhoria nos índices de cobertura dos serviços de água e esgoto no período de 1999 a 2004. Segundo a Pnad 2004, o percentual de domicílios particulares permanentes atendidos por rede geral de abastecimento de água aumentou de $80 \%$ para $83 \%$ e o percentual de domicílios servidos por esgotamento sanitário adequado (rede coletora ou fossa séptica) aumentou de $65 \%$ para $70 \%$, no referido período. Entretanto, há que se destacar as desigualdades regionais e entre municípios. Em 2005, enquanto nas regiões Sul e Sudeste, respectivamente $83 \%$ e $91 \%$ da população estavam cobertos pela rede geral de abastecimento de água, nas regiões Norte e Nordeste a cobertura desses serviços alcançava apenas $54,8 \%$ e $72 \%$ da população, respectivamente. 
No que se refere às regiões metropolitanas das capitais, as diferenças também são bastante significativas. Por exemplo, a proporção da população coberta pela rede de esgotamento sanitário, em 2005, variava de 44,26\% em Recife e 66,33\% em Fortaleza até 92,21\% em Curitiba e 91,97\% em Porto Alegre.

\section{Condições de Emprego e Trabalho}

Os problemas de saúde dos trabalhadores estão intimamente relacionados com o grau de desenvolvimento alcançado por um país ou uma região. Acompanhando as grandes diferenças no desenvolvimento social e econômico das diversas regiões do Brasil, as características da saúde dos trabalhadores e das lesões e doenças relacionadas ao trabalho mostram um padrão misto, caracterizando uma carga dupla de doenças. Verifica-se a presença de algumas doenças já controladas em países desenvolvidos, como a silicose e outras pneumoconioses, envenenamento por chumbo e asbestose, ao lado de outras "novas" doenças relacionadas ao trabalho, como afecções musculoesqueléticas, doenças dermatológicas causadas por compostos químicos, além dos sintomas e desordens mentais relacionadas ao estresse. A combinação de formas tradicionais e novas de organização do trabalho acaba por determinar altos níveis de exposição aos perigos químicos e físicos, tarefas repetitivas, excessivo uso de força, posturas inadequadas, exposição ao estresse e fatores psicossociais, causando sofrimento e incapacidades temporárias e de longo prazo .

De acordo com os dados oficiais, referentes, unicamente, aos trabalhadores formais, em 2005, 2.700 trabalhadores morreram e 491.000 ficaram fora do trabalho recebendo benefícios do seguro do trabalhador. No período de 2000 a 2002, o INSS reconheceu 58.978 casos de doenças relacionadas a trabalho, o que corresponde a uma cobertura de somente $23 \%$ dos trabalhadores, aqueles que têm contratos formais e são elegíveis para benefícios. Conseqüentemente, um grande número de casos permanece desconhecido.

\section{Ambiente e Saúde}

O impacto da poluição do ar na saúde, no Brasil, tem sido amplamente documentado na literatura. Os estudos têm mostrado que aumentos nos níveis de poluentes do ar se associam a aumentos na mortalidade e na morbidade, tanto por problemas respiratórios como cardiovasculares, em especial entre idosos e crianças. Outros efeitos referem-se a perdas econômicas, aumento no absenteísmo escolar, dias de trabalho perdidos, asma e nebulizações.

$\mathrm{Na}$ década de 1990, as primeiras estimativas de efeito da poluição do ar mostraram que a mortalidade total de idosos está diretamente associada com a variação do material particulado inalável (PM10), pois variações de $10 \mu \mathrm{g} / \mathrm{m} 3$ nas suas concentrações aumentam as mortes de idosos em 1,3\%. Entre essas mortes, a maior parte se deve às doenças respiratórias e cardiovasculares. Os efeitos dos poluentes podem ser modulados pela condição socioeconômica daqueles que estão expostos. Os indivíduos apresentam respostas diferentes a estímulos semelhantes em função das suas condições de vida. Esse ponto é de fundamental 
importância na formulação de políticas públicas voltadas para o estabelecimento de metas de redução de emissão de poluentes.

\section{Acesso a Serviços de Saúde}

Apesar de inegáveis avanços na produção de serviços e dos princípios de universalidade e eqüidade que regem o SUS, ainda se observam importantes desigualdades na oferta de recursos e serviços, assim como uma forte influência da posição social dos indivíduos no acesso, utilização e qualidade dos serviços de saúde.

Dados da Pnad de 2003 mostram que as pessoas da classe de maior renda têm 59,5\% mais chances de usar serviços de saúde do que aquelas da classe de menor renda. o mesmo efeito é observado em relação à escolaridade: entre os indivíduos com nove ou mais anos de escolaridade a chance de uso é 20,9\% maior do que a das pessoas de menor escolaridade. Apesar da persistência de um padrão de marcadas desigualdades sociais no uso de serviços de saúde, tanto para adultos quanto para as crianças, observa-se uma tendência de redução ao se comparar com as informações obtidas na Pnad de 1998.

\section{Acesso à Informação}

Um determinante social da saúde, cuja importância nem sempre é reconhecida com o destaque que merece é o acesso à informação. O acesso à informação em saúde está hoje grandemente facilitado pelas novas tecnologias de informação e comunicação (TIC), particularmente a Internet, as quais podem exercer grande influência sobre a situação de saúde e, em particular, sobre as iniqüidades em saúde. Entretanto, este potencial das TIC está ameaçado se não se resolvem as iniqüidades de acesso a essas tecnologias, também chamadas de brecha digital ou digital divide. Segundo o Ibope/Netratings, no terceiro trimestre de 2007, os usuários de Internet no Brasil, apesar de um crescimento de cerca de $21 \%$ em relação ao ano passado, são atualmente cerca de 39 milhões, uma minoria da população total, que através deste meio consegue acesso a bens e oportunidades para ascender socialmente. Dados da Pnad 2006 revelaram enormes desigualdades no acesso à Internet segundo escolaridade, renda e região de moradia, com gradientes que chegam a mais de 60 ou 70 vezes de diferença entre os dois grupos situados nos extremos de renda e escolaridade.

\subsection{Redes Sociais, Comunitárias e Saúde}

As redes sociais e comunitárias são constituintes do chamado capital social, entendido como o conjunto das relações de solidariedade e confiança entre pessoas e grupos. O desgaste do capital social é um importante mecanismo através do qual as iniqüidades socioeconômicas impactam negativamente a situação de saúde. Países com frágeis laços de coesão social resultantes dessas iniqüidades são os que menos investem em capital humano e em redes de apoio social e são também onde há menor participação na definição de políticas públicas. 
São poucos os estudos conduzidos na população brasileira que relacionam redes sociais e comunitárias com agravos em saúde, o que pode ser explicado, em parte, pela falta de domínio de metodologias adequadas para abordar estes objetos. Estudos com populações de idosos mostraram que a manutenção da independência para as atividades da vida diária, autonomia e satisfação com relacionamento familiar e amizades foram fatores preditivos independentes do envelhecimento bem-sucedido, tanto para homens como para mulheres. Estudos sobre associação entre transtornos mentais comuns e apoio social mostram que pessoas com baixo apoio social apresentaram maior prevalência de transtornos mentais comuns do que as com alto apoio social. O apoio social manteve-se associado aos transtornos mentais comuns mesmo após o ajuste por idade, escolaridade e participação no mercado de trabalho.

\subsection{Comportamentos, Estilos de Vida e Saúde}

Esta seção está baseada na revisão da literatura recente sobre dieta, exercício físico, tabagismo e alcoolismo e sua distribuição entre os diversos grupos sociais.

\section{Dieta}

O consumo de frutas e verduras é baixo, no Brasil como um todo, e quanto maior a renda e a escolaridade, maior o consumo destes alimentos. Há uma participação direta da renda no padrão de consumo: a cada $1 \%$ no aumento da renda, observa-se um aumento de 0,04\% das frutas, legumes e verduras (FLV) na composição da dieta; por outro lado, o decréscimo de 1\% no preço destes produtos aumenta sua participação na dieta em $0,2 \%$.

\section{Tabagismo}

Comparando dois inquéritos nacionais (Pesquisa Nacional de Saúde - PNS/1989 e Pesquisa Mundial de Saúde - PMS/2003), houve redução importante no hábito de fumar, em todas as faixas etárias, em ambos os sexos e no meio urbano e rural. No entanto, observam-se desigualdades na intensidade da redução, de acordo com o poder aquisitivo e a escolaridade. No caso do poder aquisitivo, o padrão foi semelhante em homens e mulheres: quanto menor a renda, menor a intensidade da redução; já para escolaridade, as diferenças foram mais acentuadas nas mulheres: quanto menor a escolaridade, menor a redução. As mulheres com escolaridade igual ou menor a quatro anos apresentaram o dobro da prevalência de tabagismo comparado com aquelas com escolaridade entre 9 a 11 anos de estudo. Na verdade, as desigualdades se acentuaram em 2003, apesar do declínio em todas as categorias.

\section{Alcoolismo}

Em um inquérito realizado pelo Instituto Nacional do Câncer (Inca), a prevalência de consumo de bebida alcoólica, nas 15 capitais brasileiras e no Distrito Federal, variou de 32,4\% a 58,6\%. A prevalência de consumo nos homens variou de $48,9 \%$ a $72,1 \%$, enquanto que em mulheres essa variação foi de $19,7 \%$ a 47,5\%, devendo-se observar que mesmo a maior taxa encontrada para mulheres $(47,5 \%)$ foi inferior a menor encontrada para homens. 
Em sociedades desenvolvidas, principalmente os países europeus, essas diferenças de gênero não são tão evidentes, em torno de $10 \%$ a $20 \%$. Nos países em desenvolvimento, essas diferenças são maiores, sendo que na América Latina, essas diferenças variaram de $20 \%$ a $100 \%$, dependendo do país. Esta variação pode ser explicada pelo tipo de bebida consumida com mais freqüência. Em Porto Alegre, na região Sul, onde se concentra a produção vinícola do país, observou-se a menor diferença por gênero (40\%).

As diferenças por gênero costumam ser ainda mais marcantes quando se avalia o consumo de risco. Entretanto, as taxas desse indicador, neste estudo, ficaram entre 4,6\% e 11,1\%. Na região Sul, apesar das taxas de consumo terem sido altas, em comparação às encontradas nas outras regiões, as taxas para consumo de risco encontraram-se entre as menores, o que deve estar relacionado às diferenças regionais no tipo de bebida consumida.

\subsection{Saúde Materno-Infantil}

Esta seção consta de dois componentes. O primeiro deles analisa os determinantes da mortalidade da infância no Brasil e regiões, destacando entre eles a renda e escolaridade da mãe. O segundo está baseado numa revisão da literatura científica sobre diversos aspectos da saúde materno-infantil no Brasil.

\section{Alguns determinantes da mortalidade na infância no Brasil}

A mortalidade na infância, ou seja, em menores de cinco anos tem sido utilizada como um bom indicador de avaliação das condições de saúde e de vida da população. Em sua fase mais recente (1990/2005), a mortalidade na infância, no Brasil, passa de 53,7 óbitos de menores de 5 anos por 1.000 nascidos vivos, para $28,8 \%$, ou seja, um decréscimo de $46,4 \%$.

Apesar dos avanços alcançados no indicador, persistem, ainda, profundos contrastes regionais. Durante o período, a região Nordeste apresentou declínios na mortalidade na infância de aproximadamente $55 \%$, ou seja, 10 pontos percentuais acima da média nacional. Todavia, o valor da taxa, nessa região, em 2005 (38,9\%), ainda representa o dobro da observada para as regiões Sudeste e Sul do país, significando uma melhoria, na medida que, em 1990, essa relação era de 2,5.

Existe uma relação inversa entre o nível de educação da mãe e a mortalidade na infância, ou seja, a medida em que aumenta a escolaridade materna, diminui de forma intensa a mortalidade de menores de 5 anos. Neste sentido, para o Brasil como um todo, em 1990, enquanto esta mortalidade, que era de $89,7 \%$ em crianças cujas mães tinham menos de quatro anos de instrução, se reduz para 30,3\% em crianças com mães com nível de instrução superior a oito anos, representando um diferencial de $196,6 \%$.

A mesma situação de desigualdade na sobrevivência de crianças se repete quando se considera o impacto de outra variável socioeconômica importante, como é o caso da renda. Esta variável, quando desagregada por quintis de renda familiar per capita, mostra que a mortalidade na infância é sempre superior para os quintis de renda familiar per capita mais pobre. De modo geral, nota-se que as diferenças entre os três primeiros quintis de renda 
familiar per capita são pequenas, acentuando-se nos dois últimos quintis, independentemente da unidade espacial analisada.

\section{Revisão da literatura sobre saúde materno-infantil}

Foi feita uma revisão da produção científica brasileira e internacional sobre os diferenciais em saúde de mães e crianças menores de cinco anos no Brasil, segundo grupos socioeconômicos e cobrindo o período de 1990 até meados de 2007.

Os resultados da revisão não deixam dúvidas sobre a presença de marcadas iniqüidades sociais em saúde e nutrição infantil em todo o país, evidenciadas nos indicadores de mortalidade, morbidade, situação nutricional e utilização de serviços de saúde. De todos os indicadores estudados, os resultados mostram que os pobres quase sempre apresentam situação menos favorável em termos de atenção pré-natal, peso ao nascer (tanto por retardo no crescimento intra-uterino como por parto pré-termo), amamentação exclusiva, cobertura vacinal, consultas preventivas, morbidade, subnutrição, deficiências de micronutrientes, desenvolvimento cognitivo e, conseqüentemente, mortalidade.

Os poucos indicadores que são piores entre as mães e crianças de famílias ricas incluem o parto por cesarianas, sobrepeso/obesidade e o uso de terapia de reidratação oral durante episódios de diarréia. Pesquisas recentes sobre amamentação mostram que esta prática, que antes era mais comum entre as crianças de famílias pobres, agora é mais comum entre as de nível socioeconômico mais elevado, pelo menos para os primeiros seis meses de vida.

\subsection{Saúde Indígena}

O mais recente censo demográfico (2000) mostra que os níveis de escolaridade dos indígenas permanecem muito baixos e que há diferenças importantes nas taxas de fecundidade total das mulheres indígenas urbanas (2,7 filhos) e rurais (5,7 filhos). Os dados censitários mostram, também, uma taxa de mortalidade infantil para os indígenas em 2000 (51,4 por mil nascidos vivos) significativamente mais elevada do que a taxa nacional (de 30,1 por mil). A mortalidade infantil indígena é muito superior a dos demais grupos de cor/raça, inclusive das crianças "pretas" e "pardas" (34,9 e 33,0 por mil, respectivamente).

As infecções respiratórias agudas e as diarréias são as principais causas de adoecimento e morte nas crianças menores de cinco anos. A desnutrição atinge mais de um quarto das crianças menores de cinco anos e, não raro, mais da metade delas. Condições precárias de saneamento e habitação, aliadas a baixa cobertura e qualidade dos serviços de saúde, interagem, levando ao agravamento e deterioração das condições nutricionais das crianças indígenas.

\section{RECOMENDAÇõES}

Esta seção inclui uma série de recomendações para intervenções sobre os DSS, com vistas a contribuir para a superação dos problemas assinalados neste relatório, particularmente para o combate às iniqüidades em saúde. 


\subsection{As Políticas e Programas em Curso}

Foi feito um mapeamento das ações de políticas sociais que têm relação com os determinantes sociais da saúde e que são executadas, principalmente, por outros ministérios que não o da Saúde, verificando sua execução orçamentária no triênio 2004-2006, e identificando as que são objeto da atuação desses vários órgãos e apresentam possibilidades ou potencialidades de articulação com o setor saúde.

Os valores e o volume de ações envolvidas indicam que o conjunto temático estudado ocupa lugar de destaque na agenda política do governo federal. O crescimento dos valores envolvidos indica que não houve, de maneira geral, depreciação nos níveis de investimento no setor.

No tocante à articulação das políticas, programas e ações, os dados sugerem: 1) baixa articulação entre os temas escolhidos, 2) baixa articulação entre os órgãos executores, 3) baixa articulação do Ministério da Saúde quanto a sua participação nas ações estudadas, 4) fragmentação das ações, 5) provável redundância de ações, 6) concorrência entre órgãos públicos federais e baixa coordenação entre estes. A despeito do caráter descritivo desta revisão, é possível adiantar algumas implicações em termos de políticas públicas: 1) ampliar o patamar de investimento das ações estudadas; 2) promover a racionalização dos investimentos, concentrando-os nas ações que apresentaram maior consistência ao longo do tempo; 3) promover a articulação dessas ações e integrar os vários órgãos federais envolvidos na execução de programas e ações, inclusive o Ministério da Saúde, em uma agenda comum pautada pelos determinantes sociais da saúde.

\subsection{A Institucionalização de Processos}

As intervenções sobre os DSS, com o objetivo de promover a eqüidade em saúde, devem contemplar os diversos níveis assinalados no modelo de Dahlgreen e Whitead, ou seja, devem incidir sobre os determinantes proximais, vinculados aos comportamentos individuais, intermediários, relacionados às condições de vida e trabalho e distais, referentes à macroestrutura econômica, social e cultural. Para que as intervenções nos diversos níveis do modelo sejam viáveis, efetivas e sustentáveis, devem estar fundamentadas em três pilares básicos: a intersetorialidade; a participação social e as evidências científicas.

Com vistas a institucionalizar um processo sustentável de coordenação das ações intersetoriais sobre os DSS, que permita superar os problemas de baixa articulação anteriormente mencionados, a CNDSS recomenda estabelecer, no âmbito da Casa Civil da Presidência da República, uma instância de Ações Intersetoriais para Promoção da Saúde e Qualidade de Vida, que deverá se responsabilizar pelo seguimento e avaliação de projetos, programas, intervenções ou políticas relacionadas aos DSS, desenvolvidas pelas diversas instituições representadas.

Essa instância deve ser coordenada pela Casa Civil da Presidência da República. O Ministério da Saúde deverá funcionar como Secretaria Técnica/Executiva. Recomenda-se que, em um primeiro momento, seja dada prioridade às ações intersetoriais relacionadas à promoção da saúde na infância e adolescência, e ao fortalecimento das redes de municípios saudáveis. Ademais, propõe-se o fortalecimento de duas outras estratégias da promoção da saúde, 
experimentadas com sucesso em diferentes contextos: as escolas promotoras da saúde e os ambientes de trabalho saudáveis.

Para a produção regular de evidências científicas sobre os DSS, sugere-se a criação de um programa conjunto MCT/MS para apoio, através de editais periódicos, a projetos de pesquisa sobre DSS e para estabelecimento de redes de intercâmbio e colaboração entre pesquisadores e gestores, visando ao seguimento dos projetos e utilização de resultados. Sugere-se, também, estabelecer um sistema de monitoramento das iniqüidades em saúde e de avaliação do impacto de ações intersetoriais sobre a saúde.

Para a promoção da participação social, com vistas a conferir a necessária base de apoio político às ações sobre os DSS e para 'empoderar' os grupos populacionais vulneráveis, sugere-se fortalecer os mecanismos de gestão participativa, principalmente os Conselhos Municipais de Saúde.

\section{ANEXO}

As atividades desenvolvidas pela CNDSS estiveram organizadas em cinco linhas de ação. No âmbito de cada uma dessas linhas, foram desenvolvidas diversas atividades que contaram com a participação de especialistas, profissionais e participantes de instituições colaboradoras, além de membros da Comissão.

A primeira linha de ação se refere à Produção e Disseminação de Conhecimentos e Informações, tendo por objetivo produzir conhecimentos e informações sobre as relações entre os determinantes sociais e a situação de saúde, particularmente as iniqüidades em saúde, com vistas a fundamentar políticas e programas.

A segunda linha de ação se refere a Politicas e Programas e teve por objetivo promover, coordenar e avaliar políticas, programas e intervenções governamentais e não governamentais sobre os DSS, realizadas em nível local, regional e nacional.

A terceira linha de ação se refere à Mobilização da Sociedade Civil e teve por objetivo chamar a atenção de diversos setores da sociedade sobre a importância dos DSS e sobre as possibilidades de atuação sobre eles.

A quarta linha de ação corresponde à Construção e Manutenção de Portal sobre DSS (www. determinantes.fiocruz.br), com o objetivo de coletar e registrar informações e conhecimentos sobre DSS, disponíveis nos sistemas de informação e na literatura científica nacional e internacional, constituindo-se em um espaço de referência para os interessados no tema.

A quinta linha de ação se refere à Cooperação Internacional, que inclui cooperação com a Comissão sobre Determinantes Sociais da Saúde da OMS (CSDH) e com os países da América Latina, para promoção do enfoque de DSS em suas políticas de saúde e/ou criação de suas respectivas comissões nacionais. 